\title{
"IL TEMPO DELLA MALAFEDE": L'ATTUALITÀ DEL PENSiero di Nicola Chiaromonte
}

\author{
Doris N. Cavallari ${ }^{*}$
}

ABSTRACT: Nicola Chiaromonte era giornalista e saggista, erede delle idee di Andrea Caffi, amico di personalità come Albert Camus e Hanna Arendt e integrante del gruppo newyorkese della Politics di Dwight Macdonald. Ha scritto innumerevoli saggi sulla società del ventesimo secolo che ci possono aiutare a capire la crisi attuale dei valori e dell'arte. Questo testo si propone a discutere alcune delle idee del saggista presenti, specialmente, nel testo 'Il tempo della malafede', nel quale l'autore parla delle "menzogne utili" che si sovrappongono alle verità "inutili" nel nostro mondo "artefatto - interamente voluto e costruito dall'uomo". Con questo saggio, Chiaromonte anticipa l'attuale concetto della "post-verità" (o post-truth, secondo l'Oxford Dictionary).

PAROLE CHIAVE: Nicola Chiaromonte; saggio; malafede; post-verità.

RESUMO: Nicola Chiaromonte era jornalista e ensaísta, herdeiro das ideias de Andrea Caffi, amigo de personalidades como Albert Camus e Hanna Arendt e parte integrante do grupo nova-iorquino da Politics de Dwight Macdonald. Escreveu inúmeros ensaios sobre a sociedade do século XX que podem nos ajudar a entender atual crise dos valores e da arte. Este texto propõe-se a discutir algumas das ideias do ensaísta presentes, especialmente, no texto Il tempo della malafede ("O tempo da má fé"), em que o autor fala das "mentiras úteis" que se sobrepõem às verdades "inúteis" do nosso mundo "artefato" - totalmente desejado e construído pelo homem". Com este ensaio Chiaromonte antecipa o atual conceito de "pós-verdade" (ou post-truth, de acordo com o Dicionário Oxford).

PALAVRAS-CHAVE: Nicola Chiaromonte; ensaio; má-fé; pós-verdade.

ABSTRACT: Nicola Chiaromonte was a journalist and an essayist, heir to the ideas of Andrea Caffi, he was also a friend of personalities like Albert Camus and Hanna Arendt and part of the New York group of the Politics, the Dwight Macdonald's review. Chiaromonte wrote countless essays on twentieth century society that can help us understand the current crisis of values and art. This text proposes to discuss some of the ideas of the 
essayist, especially in the text "Il tempo della malafede" (published in the British review Encounter as "The Will to question"), in which the author discuss the "useful lies" that overlap the "useless" truths in our "artifact world - entirely wanted and built by man". Chiaromonte, with this essay, anticipates the current concept of "post-truth" (according to the definition of the Oxford Dictionary).

KEY-WORDS: Nicola Chiaromonte; essayist; bad faith; post-truth 
Non v'è peggiore schiavitù di quella che s'ignora.

Ignazio Silone

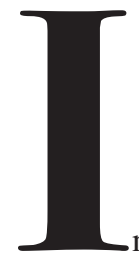

ntellettuale sensibile, giornalista e saggista legato alle questioni politiche del "Tempo presente", Nicola Chiaromonte (Rapollo - Lucania, 1905, Roma, 1972) ha scritto diversi testi per riflettere sulla nostra epoca. Spesso li ha pubblicati, modificandoli sempre un po', più di una volta. È il caso del saggio che ora commento.

L'autore, tra il 1956 e 1968 ha diretto, con Ignazio Silone, la rivista Tempo presente: informazione e discussione mantenuta dalla CCF o Congress for the Culture Freedom (In Italia, Associazione per la libertà della Cultura) fondata dai paesi alleati a Berlino, nel 1950, per divulgare la produzione culturale dei paesi detti liberi o almeno non sottomessi a un'ideologia di stato. Le riviste, infatti, davano spazio alle manifestazioni d'intellettuali di tutto il mondo, specialmente quelli che riuscivano a fuggire dall'Est europeo e avevano difficoltà di pubblicare negli stati democratici in cui la classe intellettuale era molto legata ai partiti di sinistra, come era il caso dell'Italia. Molti testi venivano pubblicati nelle varie riviste mantenute dalla CCF in diversi paesi e tradotti nella lingua della cultura locale, il che standardizzava da una parte gli ideali proposti dalla CCF e dall'altra informava il mondo "libero e democratico" sulle questioni proposte, specialmente, da autori fuggiti dai paesi comunisti.

Nell'editoriale del primo numero di Tempo presente i direttori affermano che la loro rivista vuole informare e discutere i fatti e le idee di un mondo moderno che ormai non ha un centro o confini definiti, non perché non ha più quei confini geografici o politici, ma

Perché sono incerti e problematici i confini del nostro mondo morale; incerte le norme del comportamento individuale, incerti il significato e i limiti dell'azione politica quale oggi la si pratica o la si propugna; incerto soprattutto il valore delle idee e ideologie correnti. (EDITORIALE, 1956, p. 1).

Insomma, Chiaromonte e Silone avvertono un mondo in cui ci sono 
centri di potere economico, tecnologico e politico; ma [dove] non vediamo ancora l'essenziale, ossia un centro di prestigio intellettuale e morale indiscusso e valido per tutti. In attesa, è legittimo dire che il centro, per ora, è la dove la coscienza del vero si manifesta più libera ed energica. (EDITORIALE, 1956, p.1)

"Il tempo della malafede" è proprio un testo che cerca di discutere dove "la coscienza del vero" si manifestava in quel periodo e, si può dire, si manifesta ancora oggi. Apparso inizialmente nella Rivista Volontàl, col titolo "Tempo di malafede", nel dicembre 1952 (con solo cinque pagine), l'articolo presentava sinteticamente le idee dell'autore poi rielaborate per la pubblicazione in Tempo presente (d'ora in poi TP), con il titolo 'Il tempo della malafede' (TP, ano XII, n. 4, 1967, p.6-14), per il numero 4, di aprile 1967, ossia, un mese prima della storica riunione della $\mathrm{CCF}$ che ha avuto luogo a Parigi, nella quale è stato finalmente rivelato che l'associazione, ufficialmente mantenuta dalla Fondazione Ford che sponsorizzava Centri di Cultura, biblioteche e anche la pubblicazione delle riviste, tra le quali Tempo presente, era in verità mantenuta dalla CIA. La denuncia era stata fatta dal quotidiano New York Times un anno prima (nel 1966) e la notizia è stata confermata dal nord-americano Melvin Lazky, uno dei direttori della rivista inglese Encounter, presidente della CCF, e lui stesso un agente della CIA. Il fatto ha causato molta polemica e, per la prima e unica volta, si è visto il timido Nicola Chiaromonte avanzare su Lasky in un momento di ira indignata.

Il tempo della malafede inizia così: 'La nostra non è un'epoca di fede, ma neppure d'incredulità. È un'epoca di malafede, cioè di credenze mantenute a forza, in opposizione ad altre e, soprattutto, in mancanza di altre genuine" (CHIAROMONTE, 1967, p. 6). L'autore analizza la modernità a partire dalla artificiosità che caratterizza il mondo meccanizzato, o come afferma lui stesso, un mondo

interamente artefatto - interamente voluto e costruito dall'uomo - [dove] tutto appare inconfutabile e niente naturale; anzi, l'idea stessa di naturalezza sembra la più equivoca di tutte: si applica tuttalpiù ai moti istintivi e a quella che si chiama "vita interiore". Ma moti istintivi, vita interiore hanno bisogno, per tradursi in parole e azioni schiette e veridiche, di un linguaggio che li sostenga.... Ma in un mondo artefatto e organizzato secondo necessità meccaniche, ciò che si deve credere è già stabilito di forza: è ciò cui conviene adattarsi per motivi di utilità; e, avendo accettato l'utilità come criterio, si è anche accettato che la vita consista nella ricerca dell'utile e del possesso materiale e che la questione del vero e del falso non abbia altra importanza che "teorica", vale a dire oziosa (CHIAROMONTE, 1967, p.6 - corsivo dell'autore).

1 Rivista anarchista (portavoce, a seconda dei suoi fondatori Cesare Zaccaria e Giovanna Caleffi, della "rivoluzione libertaria"), fondata a Napoli nel 1946 e pubblicata fino al 1962. 
Per Chiaromonte, nella società della malafede le "menzogne utili" sono perfettamente accettate, proprio perché "utili, facilmente utilizzabili e universalmente usate" e si sovrappongono alle "verità inutili", quelle che non hanno un'utilità pratica e immediata.

Una società che vive sotto le "menzogne utili" non cambia inizialmente il modo di agire di ogni individuo, ma "cambia lo stato della società. Il quale, a sua volta, mutando, certamente altera, se non la natura dell'individuo, almeno la forma dei suoi rapporti con gli altri." (CHIAROMONTE, 1967, p.7) In queste società, anche gli uomini giusti saranno influenzati dai valori dubbi, perché è impossibile rimanere incolumi a una logica sociale perversa che invade la vita dell'individuo

è impossibile immaginare che l>individuo riesca a rimanere immune da costrizione e falsificazione in una società in cui la forma stessa dei rapporti sociali - ossia i modi della comunicazione - siano falsati e corrotti (CHIAROMONTE, 1967, p.7)

perché la società non è una collezione d'individui o delle istituzioni politiche e giuridiche e economiche che la reggono, ma è "l'insieme delle credenze secondo le quali i membri di una comunità si accordano o vengono in conflitto" (CHIAROMONTE, 1967, p.7), cioè la società è fatta di relazioni e l'uomo deve in qualche modo adattarsi a certi valori.

Vorrei soffermarmi su questa affermazione sull'accordo o conflitto dei membri di una comunità e portare alla discussione alcune questioni riunite dalla giornalista britannica Frances Saunders nel libro Who paid the piper? The CIA and the Culture Cold War, del 1999 (In italiano, La guerra fredda culturale: la Cia e il mondo delle lettere e delle arti). Saunders propone un percorso storico della CCF e delle sue riviste e commenta alcune azioni fondamentali per capire la politica della CCF/CIA per evitare i conflitti tra i membri dell'Associazione che parlavano per il "mondo libero e democratico". Si portava avanti, infatti la politica "di mentire per la verità" e delle "menzogne utili" di cui parla Chiaromonte (che l'autrice cita spesso nel suo libro) praticata specialmente dalla rivista Encounter, la portavoce della CCF in Inghilterra e la più prestigiosa di tutte le riviste, insieme alla Preuves francese. È noto, per esempio, il caso della censura della Encounter che ha impedito la pubblicazione del testo "America, America!" del polemico giornalista Dwight Macdonald, che al suo ritorno da un soggiorno in Europa, si è turbato con i valori nordamericani e la mancanza di raffinamento dei suoi connazionali.

Così, mentre la CCF s'impegnava a denunciare l'oppressione del regime comunista usava, in difesa della libertà dell'Occidente, il silenzio e l'omissione sui problemi del "mondo libero", specialmente quelli nordamericani. Chiaromonte, però, aveva già scritto come editore invitato per il secondo numero di Encounter, nel 1953, il testo The Will to question, infatti, la versione inglese, un po' mofidicata, del “Tempo di malafede" pubblicato su Volontà nel 1952, nel quale l'autore, come riporta Saunders insiste che "Il dovere a cui nessun intellettuale può sottrarsi senza degradare se stesso è il dovere di denunciare le menzogne e di rifiutarsi di chiamare verità le 'utili bugie"” (CHIAROMONTE, 1952, p. 557) Ma, osserva Saunders, mentre Encounter 
insisteva sulle denunce delle utili bugie con le quali i regimi comunisti cercavano di sostenersi, non si è mai liberata completamente della "trappola dell'ideologia", dalla pervasiva psicologia della guerra fredda, del "mentire per la verità".(SAUNDERS, 2004, p. 287)

Analizzando la storia recente, Chiaromonte afferma che il tempo della malafede è cominciato esattamente il 2 agosto del 1914, proprio il giorno in cui è iniziata la Prima Guerra mondiale, in cui la fede del progresso umano basato sulla scienza e sulla ragione è stato sconfitto. Ma per il polemico giurista tedesco, Carl Schimtt, la data sarebbe il 2 aprile 1917 quando gli Stati Uniti decisero di entrare in guerra contro la Germania. Danilo Zolo nella prefazione al libro Il processo discriminatorio di guerra, di Schmitt, tradotto e pubblicato da Laterza nel 2008, riassume le idee dell'autore tedesco sull'argomento. Per Schmitt, con l'entrata in guerra, gli Stati Uniti hanno rotto la propria politica di neutralità per "garantire la libertà dei popoli e la pace mondiale" (ZOLO, 2008, p. V) fatto che ha determinato una svolta nella politica mondiale e che ha delineato con chiarezza un triplice fenomeno:1) L'emergere definitivo degli Stati Uniti d'America come potenza fautrice di un nuovo imperialismo e, di conseguenza, la fine della centralità politica e giuridica dell'Europa; 2) il tramonto dello jus publicum europaeum quale strumento di regolazione della guerra fra Stati, e il profilarsi di istituzioni internazionali "universalistiche" - anzitutto la Società delle Nazioni - che avrebbero preteso di garantire la pace attraverso la prescrizione giuridica della guerra; 3) l'avvento di una guerra globale "discriminante", poiché entrando in guerra contro la Germania, gli Stati Uniti avevano annullato i concetti non discriminatori di guerra e di neutralità e si erano attribuiti il potere di decidere su scala internazionale quale parte belligerante avesse ragione e quale torto.

Sarebbe lungo discutere le idee di Schmitt, però, il fatto è che dalla Prima Guerra mondiale in poi, il mondo verrà diviso un due blocchi politico-ideologici di grandi potenze a cui tutti i paesi meno influenti si sarebbero dovuti sottomettere. Saunders rifà i passi della Cia e la sua politica di difesa del mondo "libero", citando documenti dell'IOD (International Organizations Division e del National Security Council, la NSC-68), abbozzati nel marzo 1950, ossia, alcuni mesi prima della fondazione della CCF avvenuta nel luglio dello stesso anno e commenta la conclusione del documento guida della NSC- 68:

"Considerazioni ideologiche e pratiche [...] ci impongono la conclusione che non abbiamo altra scelta che dimostrare la superiorità dell'idea di libertà attraverso la sua applicazione costruttiva". "Anche la verità ha bisogno di propaganda», aveva recentemente affermato il filosofo Karl Jaspers. Questo era il mandato che autorizzava i combattenti americani della guerra fredda a prendere misure "costruttive" per assicurarsi che la verità trionfasse sull'inganno.”(SAUNDERS, 2004, p. 89-90)

Saunders cita ancora la frase dell'assistente del segretario di Stato nord-americano Edward Barrett che afferma che "Nella battaglia per la conquista delle menti, la verità può essere la 
specifica arma americana" e conclude la giornalista britannica: "La verità, e il secolo stesso, dovevano appartenere all'America. Se si doveva ricorrere all'inganno per promuovere la verità, bene, lo si sarebbe fatto."(SAUNDERS, 2004, p. 90)

"L'inganno", allora, sarebbe tutto quello che veniva dal mondo comunista. Così "il tempo della malafede" vede nascere due forze politiche antagonistiche: quella democratica liberale dei paesi alleati e quella del socialismo sovietico; ambedue celano sotto la propaganda ragioni politiche, ma praticavano la manipolazione delle informazioni, la censura e il silenzio dei propri insuccessi.

Chiaromonte analizza la malafede come questa tendenza al nichilismo caratteristico del mondo moderno che richiede una corrente "vincente" che conduca le società a credere a un'egemonia del vincitore e si traduce in una "volontà egomaniaca di dominio e di 'totalizzazione' del mondo in un'unica idea" (CHIAROMONTE, 1967, p.12) e in "un'empietà radicale, in quanto esso porta a ignorare tutto ciò che non serve a fini immediati e a negare con ciò tutto quello che nel mondo è intimo, indicibile, arcano" (CHIAROMONTE, 1967, p.13).

Alla fine del suo testo Chiaromonte ci invita a liberarci dalla "credulità nel mondo attuale e nei suoi idoli", dall'idea che il corso delle cose dovrebbe avere un significato unico e, ancora, dal falso ottimismo che regola la società secondo il valore della produzione e ci invita a cercare valori per dare nuovi significati alla vita umana (CHIAROMONTE, 1967, p.14)

Nel 2016 l'Oxford Dictionary ha eletto il lessema post-truth (o post-verità) come la parola dell'anno, legandola al fenomeno del "brexit" e alle elezioni del presidente nord-americano Donald Trump. Interessante notare che la parola nasce dalle politiche dei due grandi paesi anglofoni. Sulla pagina dell'Accademia della Crusca, la post-verità è definita come parola "rimbalzata dai giornali al web e viceversa: "relativo a, o che denota, circostanze nelle quali fatti obiettivi sono meno influenti nell'orientare la pubblica opinione che gli appelli all'emotività e le convinzioni personali”. (BIFFI, 2016, on-line)

Tale definizione ci fa pensare a quello che avvertivano intellettuali come Nicola Chiaromonte, visto che "post-verità" è una forma attuale per definire quelle bugie necessarie sulle quali si trattiene a lungo Frances Saunders nel suo libro, nel quale osserva che

Il processo democratico che i combattenti occidentali della guerra fredda culturale si erano precipitati a legittimare era minato da questa mancanza di sincerità. La "libertà" che offrivano era compromessa, "non libera", nel senso che era basata sull'imperativo contraddittorio della "bugia necessaria“. Nel contesto della guerra fredda, come descritto da gran parte degli intellettuali militanti nel Congresso per la libertà della cultura, si operava sotto il segno di una completa fedeltà a un ideale. I fini giustificavano i mezzi, anche se ciò comportava raccontare menzogne (dirette o per omissione) a un collega; l'etica era soggetta alla politica. (SAUNDERS, 2004, p. 371) 
Le menzogne utili cercano di eliminare la percezione popolare dei pericoli della propaganda, della manipolazione politica e dell'uso efficace dei discorsi persuasivi che invitano gli uomini a credere senza ragionare. Il libro di Saunders, del 1999 e i testi di Chiaromonte sembrano più attuali che mai e sono un punto di riferimento per farci pensare in modi di incontrare nuovi valori che facciano cadere in disgrazia parole e atti che si traducono contermini come post-verità. 


\section{Riferimenti Bibliografici}

BIFFI, M. Viviamo nell'epoca della post-verità? In: Accademia della Crusca. Sezione Língua Italiana. 25 nov. 2016. Disponível em: http://www.accademiadellacrusca.it/it/lingua-italiana/consulenza-linguistica/domande-risposte/viviamo-nellepoca-post-verit. Acesso em: 28/10/2017.

CHIAROMONTE, N. Il tempo della malafede. In: Tempo Presente: informazione e discussione. Anno XII, n. 4, aprile, 1967, p. 6-14.

EDITORIALE. In: Tempo Presente. Anno I, no 1, 1956, p. 1-2.

SAUNDERS, F.S. La guerra fredda culturale. Trad. Silvio Calzavarin. Roma: Fazi Editore, 2004.

ZOLO, D. Prefazione. In: SCHMITT, C. Il processo discriminatorio di guerra. Bari-Roma: Laterza, 2008, p. V-VII.

Recebido: $15 / 06 / 2018$

Aprovado: 20/08/2018 